\title{
Comparación de la calidad de vida en estudiantes universitarios según nivel de actividad física
}

\author{
Comparison of quality of life in univ ersity students with different levels of physical activity \\ Yeny Concha-Cisternas ${ }^{1 *}$ orcid.org/0000-0001-7013-3894 \\ Marcelo Castillo-Retamal2,3 orcid.org/0000-0002-7482-1165 \\ Eduardo Guzmán-Muñoz ${ }^{4}$ orcid.org/0000-0001-7001-9004
}

\begin{abstract}
Univ ersidad Tecnológica de Chile INACAP. Santiago, Chile
Departamento de Ciencias de la Activ idad Física, Universidad Católica de Maule, Talca, Chile

Grupo de Investigación en Activ idad Física y Salud, GEAFyS, Univ ersidad Católica de Maule, Talca, Chile

Escuela de Kinesiología, Facultad de Salud, Universidad Santo Tomás, Santiago, Chile
\end{abstract}

Fecha de recepción: Febrero 25 - 2019

Fecha de rev isión: Septiembre $03-2019$

Fecha de aceptación: Diciembre 19 - 2019

Concha-Cisternas Y, Castillo-Retamal M, Guzmán-Muñoz E. Comparación de la calidad de vida en estudiantes universitarios según nivel de actividad física. Univ. Salud. 2020;22(1):33-40. DOI: https://doi.org/10.22267/rus.202201.172

\section{Resumen}

Introducción: Los universitarios son susceptibles a estilos de vida poco saludables, los que podrían deterior su calidad de vida (CV). Objetivo: Comparar la CV en estudiantes universitarios según su nivel de actividad física (AF). Materiales y métodos: Estudio descriptivo, transversal. Participaron 126 estudiantes universitarios de ambos sexos de las facultades de salud, educ ación e ingeniería de tres universidades de Talca (Chile). La CV fue evaluada mediante el cuestionario WHOQOL-BREF. Se aplicó la $t$ de student para muestras independientes para comparar los dominios de la CV y las características basales entre los grupos. El tamaño del efecto se calculó con la d de Cohen. Resultados: Los universitarios inactivos presentaron mayor índice de masa corporal (IMC) $(p=0,041)$, en contraste, los universitarios activos mostraron una puntuación significativamente may or que los inactivos en CV global $(p=0,002 ; d=0,67)$, CV en salud $(p=0,013 ; \mathrm{d}=0,50)$, dominio físico $(p=0,038 ; \mathrm{d}=0,43)$, psicológico ( $p=0,003$; $d=0,63)$ y medio ambiente $(p=0,001 ; d=0,80)$. Conclusiones: Los estudiantes universitarios activos (moderado/alto nivel de AF) presentan mejor CV global y en salud, además presentan mejor puntuación en CV en los dominios físico, psicológico medio ambiente al compararlos con estudiantes inactivos.

Palabras clave: Actividad física; salud; estudiantes; calidad de vida; estilo de vida. (Fuente: DeCS, Bireme).

\begin{abstract}
Introduction: University students are susceptible to unheal thy lifestyles, which could deteriorate their quality of life (QL). Objective: To compare the QL in university students who have different levels of physical activity (PA). Materials and methods: A descriptive, cross-sectional study that included 126 university students of both sexes from Health, Education and Engineering programs of three universities in Tal ca (Chile). The QL was assessed through the WHOQOL-BREF questionnaire. A student's t-test of independent samples was used to compare both the domains of the QL and the baseline characteristics between the groups. The effect size was calculated with Cohen's d. Results: Inactive university students had a higher body mass index (BMI) $(p=0.041)$. In contrast, active students showed significantly higher scores than inactive ones in overall QL $(p=0.002 ; d=0.67)$, health QL $(p=0.013 ; d=0.50)$, physical domain $(0.038 ; d=0.43)$, psychological domain $(p=0.003 ; d=0.63)$ and environment domain $(p=0.001 ; d=0.80)$. Conclusions: Active university students (with moderate and high PA levels) have better overall and heal th $\mathrm{QL}$. They also have better QL scores in the physical, psychological and environmental domains when compared to inactive students.
\end{abstract}

Key words: Motor activity; health; students; quality of life; lifestyle. (Source: DeCS, Bireme).

\footnotetext{
*Autor de correspondencia

Yeny Concha-Cisternas

e-mail: yenyf.concha@gmail.com
} 


\section{Introducción}

En los últimos 30 años los países latinoamericanos han sufrido cambios nocivos sobre los estilos de vida y el nivel de actividad física (AF) ${ }^{(1)}$. En el mundo se estima que un $31 \%$ de la población general no cumple las recomendaciones de AF sugeridas por el Colegio Americano del Deporte (150 minutos de AF moderada o 75 minutos de AF vigorosa)(2), mientras que un $80 \%$ de los adolescentes es considerado físicamente inactivo(3). Similar situación ocurre en Chile, donde los datos preliminares de la Encuesta Nacional de Salud (2016-2017) muestran que un $73,5 \%$ de los jóvenes entre 15 y 19 años no realizan AF durante 30 minutos, 3 o más veces por semana ${ }^{(4,5) \text {. }}$

La adolescencia se caracteriza por ser una etapa de desarrollo susceptible a cambios en los estilos de vida, influenciados por el entorno social y familiar(6). Bajo este contexto, los estudiantes universitarios debido a largas jornadas académicas, serían una población vulnerable, ya que presentan inadecuados hábitos alimenticios, dedican un elevado número de horas a actividades de tipo sedentaria (ver televisión o actividades de transporte, trabajo y estudio), mantienen hábito tabáquico y se desplazan en transporte motorizado, lo que incrementa la inactividad física $(1,5,7)$. Todas las conductas mencionadas previamente podrían afectar su salud general y con ello, su calidad de vida (CV).

En contraste, se ha propuesto ampliamente que practicar AF de manera regular y sostenida es un importante predictor de salud mental y física, mejorando el bienestar y $\mathrm{CV}$ de las personas. Fomentar la realización de AF en jóvenes universitarios permitiría generar hábitos saludables que garanticen una mejor $\mathrm{CV}$, además programas de AF que influyan positivamente en la salud de los futuros adultos y con ello, disminuir los elevados costos de salud pública que ocasiona las enfermedades crónicas no transmisibles(8).

La Organización Mundial de la Salud (OMS) define CV como "la percepción del individuo de su posición en la vida, en el contexto cultural y el sistema de valores en que vive, en relación con sus metas, objetivos, expectativas, valores y preocupaciones $(9,10) "$. Uno de los instrumentos más utilizados para evaluar la CV corresponde al cuestionario World Health Organization Quality of Life en su versión breve (WHOQOL-BREF). Este cuestionario tiene 26 ítems y ha sido reconocido por su alta estructura conceptual, operativa y desarrollo psicométrico, siendo validado y aplicado en diferentes grupos etarios, países e idiomas(11,12). Si bien se ha propuesto que comportamientos saludables como practicar AF se asocian con mayor $\mathrm{CV}$, bienestar y satisfacción(13), en estudiantes universitarios latinoamericanos la evidencia es escasa, existiendo pocos antecedentes sobre la realidad local. Es por esto que resulta de interés comparar la CV de acuerdo al nivel de AF en estudiantes universitarios y conocer si existen diferencias en la CV al agruparlos por facultad.

Por lo tanto, el objetivo de este estudio fue comparar la $C V$ en estudiantes universitarios según su nivel de $\mathrm{AF}$.

\section{Materiales y métodos}

Esta investigación corresponde a un estudio de tipo descriptivo, de diseño observacional y de corte transversal, desarrollado en los meses de abril y junio del año 2018. Los participantes de este estudio pertenecían a las facultades de salud, educación (a excepción de estudiantes de educación física) e ingeniería de tres universidades de la ciudad de Talca (Chile) y fueron seleccionados de manera no probabilística por conveniencia. La muestra total fue constituida por 126 estudiantes universitarios (53,2\% hombres, $46,8 \%$ mujeres): 74 participantes inactivos y 52 participantes activos según su nivel de AF. La muestra corresponde al $5,4 \%$ de total de la población objetivo $(\mathrm{N}=2336)$.

Los criterios de inclusión utilizados fueron: contar con matrícula vigente durante el periodo en que fueron obtenidos los datos, tener $\geq 18$ años, aceptar participar voluntariamente del estudio y firmar un consentimiento informado que autoriza el uso de la información con fines de investigación científica. La confidencialidad de sus antecedentes, metodología y objetivo del estudio fueron explicados previo al registro de datos. Fueron excluidos los estudiantes que al momento de la recolección de datos tenían licencia médica o presentaran discapacidad física.

\section{Procedimientos e instrumentos}

Las variables de estudio fueron: antecedentes personales, estado nutricional, perímetro de cintura (PC), CV y nivel de AF. Como antecedentes personales se consideraron edad y género. Para medir el peso corporal se utilizó una balanza 
electrónica portátil (Seca 769, Alemania; precisión $0,1 \mathrm{~kg}$ ) y la estatura se midió en posición bípeda utilizando un estadiómetro portátil (Seca 217, Alemania; precisión 0,1 cm). El estado nutricional se determinó a través del índice de masa corporal (IMC) según los criterios de la OMS, que señala dividir el peso por la estatura al cuadrado $\left(\mathrm{kg} / \mathrm{m}^{2}\right)^{(14)}$. Los sujetos voluntarios fueron evaluados bajo condiciones normales de temperatura $y$ humedad. Este procedimiento lo realizó un profesional capacitado para valoración antropométrica.

El PC sugerido para población chilena fue utilizado para definir la clasificación de obesidad central (> $87 \mathrm{~cm}$ para hombres y $>82 \mathrm{~cm}$ para mujeres), para ello se utilizó una cinta métrica (Sanny, Brasil; precisión $0,1 \mathrm{~cm}$, rango $0-200 \mathrm{~cm}$ ) con el individuo en bipedestación y tomando como referencia anatómica el punto medio entre la cresta iliaca y la última costilla(14). Todas las mediciones se realizaron a una temperatura de $24^{\circ} \mathrm{C}$ y una humedad relativa del $50 \%$.

La CV fue medida a través del WHOQOL-BREF. Este es un cuestionario auto administrado, el cual contiene 26 preguntas agrupadas en 4 ítems o dominios: físico (dolor, energía, sueño, movilidad, actividades, medicación y trabajo), psicológico (sentimientos positivos, pensamiento, estima, cuerpo, sentimientos negativos y espiritualidad), social (relaciones sociales, soporte social y sexualidad) y medio ambiente (seguridad, hogar, finanzas, servicios, información, ocio, medio ambiente y transporte). Además de 2 preguntas globales: CV global y CV en salud. Cada ítem tiene 5 opciones de respuesta ordinales tipo likert, en la cual la más baja percepción es el 1 y la más alta el 5. La puntuación obtenida en los dominios fue escalada en una dirección positiva de 0 a 100 , es decir, los puntajes más elevados indican una mejor autopercepción de CV(12).

Con respecto a las propiedades psicométricas del WHOQOL-BREF este ha reportado una confiabilidad de 0,88 para el de médicos APS cuestionario total, y para cada una de las preguntas entre 0,87-0,88, demostrando con esto, que las preguntas y sus respectivas dimensiones están relacionadas y aportan una descripción apropiada de la CV de la población estudiada(15). Asimismo el cuestionario presenta una validez cercana al 0,99(15).
El nivel de AF se midió a través del Cuestionario Internacional de Actividad Física (IPAQ) versión corta, el cual consta de 7 preguntas donde incluye actividades laborales, físico-deportivas, de transporte (caminar) y el tiempo que la persona permanece sentada o recostada(13). La AF total fue expresada de manera continua en METs (Metabolicenergy-equivalents). A partir de esto, quienes presentaron un gasto energético $<600$ Mets/min/semana fueron clasificados con un bajo nivel de $A F^{(16)}$ e incluidos en el grupo denominado "inactivos". Los estudiantes que reportaron un gasto energético >600 Mets/min/semana fueron considerados con un nivel de AF moderado/alto(16), siendo clasificados en el grupo denominado "activos". En cuanto a las propiedades psicométricas, el IPAQ ha demostrado una confiabilidad de 0,8 y una validez de $0,67(17)$.

\section{Análisis estadístico}

Los datos fueron analizados con el software estadístico SPSS 23.0 (SPSS 23.0 para Windows, SPSS Inc., IL, USA). Se calculó la media y desviación estándar (DE) para describir las variables. La distribución y homogeneidad de varianza de los datos fueron determinadas con las pruebas de Shapiro-Wilk y Levene, respectivamente. Se aplicó la prueba T de Student para muestras independientes a fin de comparar los dominios de la $\mathrm{CV}$ y las características basales entre individuos activos e inactivos tanto para la muestra total como para los grupos separados por facultades. El tamaño del efecto se calculó con la $d$ de Cohen, considerando un efecto pequeño $(0,2)$, moderado $(0,5)$ o fuerte $(0,8)$. Para comparar la CV entre facultades en universitarios activos e inactivos se utilizó la prueba de ANOVA. Para todos los análisis se empleó un nivel alfa de 0,05 .

\section{Consideraciones éticas}

Esta investigación fue aprobada por el Comité de Ética de la Universidad Santo Tomás (folio 79-18), quien revisó que los procedimientos de la investigación siguieran las consideraciones éticas de la declaración de Helsinki.

\section{Resultados}

En la Tabla 1 se observan las características basales de la muestra. La diferencia observada entre los grupos revela que los estudiantes inactivos presentaron un mayor IMC $(p=0,041)$. De acuerdo con estos valores, los estudiantes inactivos se 
clasifican en la categoría de sobrepeso, mientras que los universitarios activos se clasifican como normopeso. Si bien los resultados no mostraron diferencias estadísticamente significativas para el PC $(p=0,703)$, existe una tendencia favorable hacia aquellos que poseen niveles más altos de AF.

Al comparar la $\mathrm{CV}$ a partir del cuestionario WHOQOL-BREF, los universitarios activos mostraron una puntuación significativamente mayor que los estudiantes clasificados como inactivos tanto en la CV global $(p=0,002 ; d=0,67)$ como en la CV en salud $(p=0,013 ; d=0,50)$. Al analizar la CV por dimensiones, los resultados reflejaron una mayor puntuación en los dominios físico $(p=0,038 ; d=0,43)$, psicológico $(p=0,003 ; d=0,63)$ y medio ambiente $(p=0,001$; $d=0,80$ ) en los universitarios activos (Tabla 2). Al comparar la CV entre hombres y mujeres no hubo diferencias significativas en todas las dimensiones evaluadas $(p<0,05)$.

La Figura 1 muestra la $\mathrm{CV}$ de los estudiantes divididos por áreas de estudio (ingeniería, salud y educación) y nivel de AF. Al comparar la CV en estudiantes del área de ingeniería según su nivel de $\mathrm{AF}$, se observaron puntuaciones significativamente mayores en la CV global $(p=0,011), C V$ en salud $(p=0,047)$, y en los dominios físico $(p=0,042)$, psicológico $(p=0,001)$ y medio ambiente $(p=0,001)$ en los universitarios activos. En el área salud, los estudiantes activos presentaron puntuaciones significativamente mayores en la CV que los universitarios inactivos en todas las dimensiones evaluadas: CV global $(p=0,002), \mathrm{CV}$ en salud $(p=0,017)$, dominio físico $(p=0,001)$, dominio psicológico $(p=0,008)$, dominio social $(p=0,123)$ y dominio medio ambiente $(p=0,001)$. Finalmente, los estudiantes del área educación sólo mostraron diferencias significativas en la CV general $(p=0,043)$ $y$ en el dominio psicológico $(p=0,031)$ entre universitarios inactivos y activos.

El análisis de ANOVA reveló que al comparar la CV entre los estudiantes de las tres facultades evaluadas no hubo diferencias significativas tanto en el grupo activo como en el inactivo.

Tabla 1. Características basales de los estudiantes universitarios según el nivel de actividad física

\begin{tabular}{|c|c|c|c|c|}
\hline Variables & $\begin{array}{c}\text { Inactivos }(n=74) \\
\text { Media } \pm D E\end{array}$ & $\begin{array}{c}\text { Activos }(n=52) \\
\text { Media } \pm D E\end{array}$ & Valor $\mathbf{p}$ & Tamaño del efecto \\
\hline Edad (años) & $20,96 \pm 2,77$ & $20,83 \pm 2,03$ & 0,811 & 0,05 \\
\hline Peso (Kg) & $67,97 \pm 15,31$ & $66,48 \pm 10,68$ & 0,865 & 0,11 \\
\hline Talla (m) & $1,64 \pm 0,09$ & $1,66 \pm 0,09$ & 0,402 & 0,00 \\
\hline IMC (Kg/m2) & $25,42 \pm 4,59$ & $24,12 \pm 3,60$ & 0,041 & 0,31 \\
\hline$P C(\mathrm{~cm})$ & $83,88 \pm 12,66$ & $80,17 \pm 9,55$ & 0,703 & 0,33 \\
\hline ICC $(\mathrm{cm})$ & $0,80 \pm 0,07$ & $0,80 \pm 0,07$ & 0,690 & 0,00 \\
\hline
\end{tabular}

IMC: Índice de Masa Corporal

PC: Perímetro de Cintura

ICC: Índice Cintura Cadera

Tabla 2. Resultados de la comparación de calidad de vida entre universitarios con bajo y alto nivel de actividad física

\begin{tabular}{|c|c|c|c|c|}
\hline & $\begin{array}{c}\text { Inactivos }(n=74) \\
\text { Media } \pm \text { DE }\end{array}$ & $\begin{array}{c}\text { Activos }(n=52) \\
\text { Media } \pm \text { DE }\end{array}$ & Valor $p$ & Tamaño del efecto \\
\hline CV Global & $3,12 \pm 0,94$ & $3,72 \pm 0,83$ & 0,002 & 0,67 \\
\hline CV en Salud & $3,10 \pm 1,08$ & $3,65 \pm 1,08$ & 0,013 & 0,50 \\
\hline Dominio físico & $66,11 \pm 10,14$ & $71,27 \pm 13,59$ & 0,038 & 0,43 \\
\hline Dominio Psicológico & $67,90 \pm 10,33$ & $74,87 \pm 11,71$ & 0,003 & 0,63 \\
\hline Dominio Social & $69,45 \pm 14,96$ & $75,64 \pm 14,14$ & 0,058 & 0,42 \\
\hline Dominio Medio ambiente & $66,71 \pm 12,24$ & $76,00 \pm 10,72$ & 0,001 & 0,80 \\
\hline
\end{tabular}

DE: desviación estándar; CV: calidad de vida 


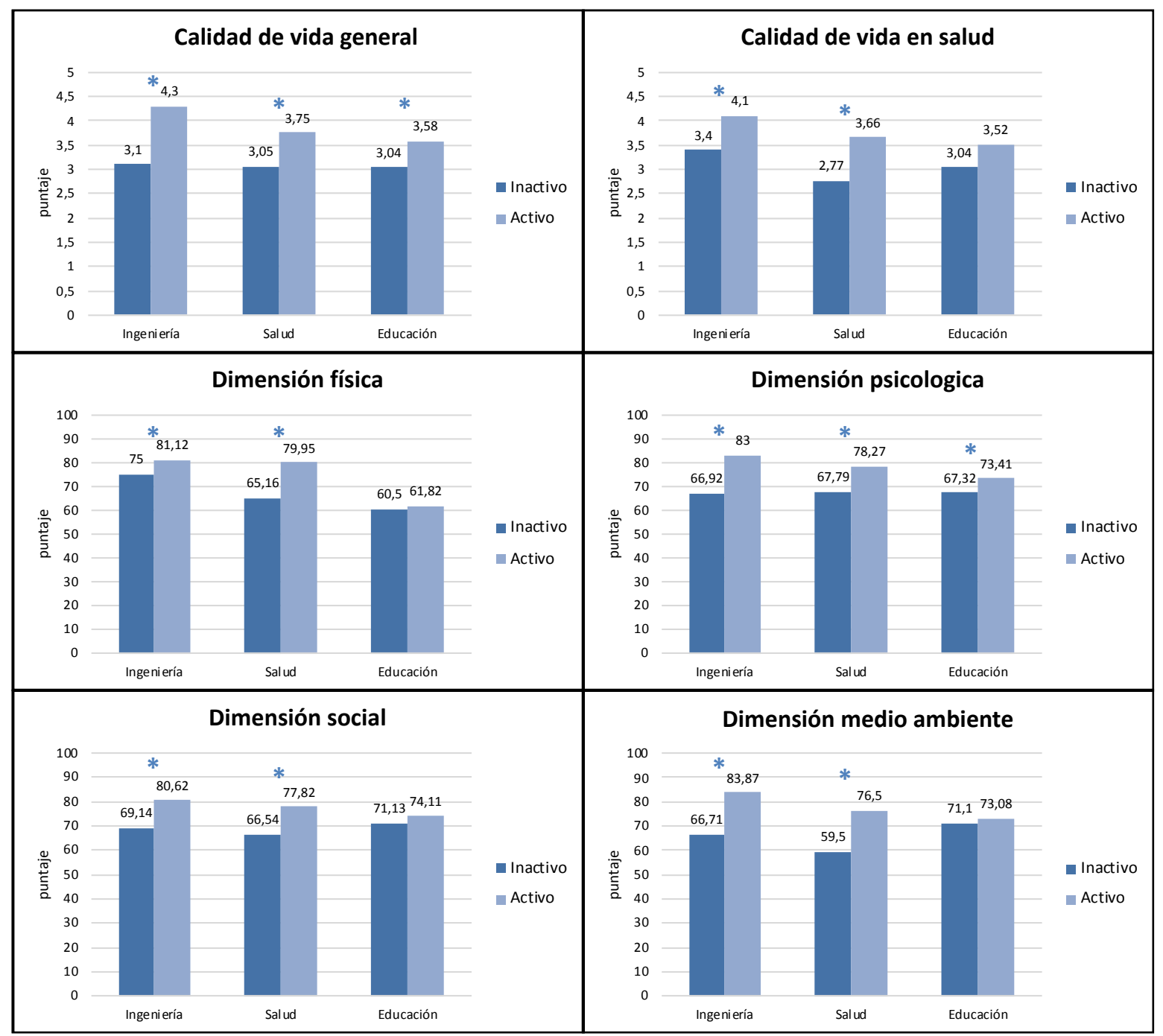

*Diferencias estadísticamente significativas $(p<0,05)$

Figura 1. Comparación de los dominios de calidad de vida según nivel de actividad física

\section{Discusión}

El principal resultado de esta investigación señala que los estudiantes que practican regularmente $\mathrm{AF}$ presentan una mejor CV que sus pares inactivos. Estas diferencias se observaron en la CV global y en salud, y en los dominios físico, psicológico y medio ambiental. Similares resultados fueron reportados en estudiantes universitarios mexicanos, donde se observó una asociación positiva entre la práctica regular de $\mathrm{AF}$ con una mejor $\mathrm{CV}$, principalmente, en la CV global y en el dominio medio ambiental(18).

Asimismo, estudiantes brasileños con altos niveles de $\mathrm{AF}$ presentaron una buena $\mathrm{CV}$ en los cuatro dominios, obteniendo mayor puntuación en el dominio psicológico y un puntaje más bajo en dominio social(19). Diversos estudios señalan que la personas que siguen las recomendaciones establecidas por la OMS y el Colegio Americano del deporte sobre la práctica de 30-59 minutos/día de $\mathrm{AF}$ de intensidad moderada presentan una mejor $\mathrm{CV}(20)$.

La evaluación de la CV por dominios posibilita el conocimiento de los aspectos individuales, en los cuales una persona o grupo, presenta falencias o disconformidad. El presente estudio encontró que los universitarios activos mostraron puntuaciones más altas que los inactivos en los dominios físico, medio ambiente y psicológico. Similares hallazgos se han descrito en población adulta, en donde quienes practicaban deporte o $\mathrm{AF}$ regular, tuvieron 
puntuaciones más altas en los dominios físico, psicológico y medio ambiente, en comparación con los que eran sedentarios. Esto podría atribuirse a que la práctica de $\mathrm{AF}$ promueve un mejor autoconcepto físico y niveles de satisfacción con la vida, lo que repercute sobre el estado de ánimo de los sujetos (21).

Asimismo, se ha reportado que la práctica de $\mathrm{AF}$ estimula la activación del sistema de opioides endógenos, induciendo un incremento significativo de la concentración de $ß$-endorfinas, que se traduce en sensación de bienestar, analgesia, relajación y disminución de la ansiedad y del estrés(22,23). Dicho estado de bienestar inducido por AF también ayudaría a mejorar la percepción relacionada a las condiciones ambientales, como transporte, seguridad y cuidados con la salud, logrando de este modo mejores puntuaciones en el dominio medio ambiente(24).

$\mathrm{Al}$ analizar la muestra por facultades, la tendencia a mantener una mejor CV en los dominios evaluados se mantuvo, siendo los universitarios físicamente activos de la facultad de salud e ingeniería quienes mostraron una mayor diferencia respecto a los inactivos. Por su parte, los estudiantes de la facultad de educación evidenciaron diferencias solo en CV general y dominio psicológico. Sin embargo, al comparar los dominios de CV entre facultades tanto en grupo de activos como inactivos no se evidenciaron diferencias significativas. A pesar de que no existen reportes previos, es posible que los individuos de la facultad de educación muestren menores diferencias en la CV entre estudiantes activos e inactivos debido a mayores posibilidades de integración social que les brinda su malla curricular en los primeros años de estudio, lo cual podría influir en que individuos inactivos tengan una mejor percepción de algunos dominios de la CV evaluada, como, por ejemplo, el social y ambiental.

A pesar de los beneficios atribuidos a la práctica de $\mathrm{AF}$, la puntuación en el dominio social no fue influida por el nivel de AF de los estudiantes universitarios, lo cual podría ser explicado por el tipo de preguntas que se orientan a la satisfacción de relaciones con amigos y su vida sexual. Se ha señalado que los estudiantes reducen drásticamente el tiempo que dedican a sus familiares, pareja y amigos debido a la carga académica asociada a cada carrera(25). Esto podría ser la causa del deterioro de la CV en dicho aspecto, independiente del nivel de AF del estudiante.

Finalmente, un resultado secundario de esta investigación muestra que los estudiantes universitarios activos, tienen IMC más bajo (IMC>24,12 kg/m²) al compararlos con sus pares inactivos (IMC $25,42 \mathrm{~kg} / \mathrm{m}^{2}$ ). Estudios previos han demostrado una alta prevalencia de exceso de peso (IMC> $25 \mathrm{~kg} / \mathrm{m}^{2}$ ) en universitarios chilenos, atribuido principalmente a sedentarismo(26). En contraste, la práctica de $\mathrm{AF}$ reduce algunos índices antropométricos como el PC y el IMC (27). Si bien el IMC es reconocido como un indicador de salud cardiovascular, se ha señalado que no está relacionado solamente con problemas físicos como limitación funcional y dolor, sino que también se ha relacionado directamente con una menor $\mathrm{CV}$ en adolescentes(8).

\section{Conclusiones}

Los estudiantes universitarios activos (mantienen un moderado/alto nivel de $\mathrm{AF}$ ) presentan una mejor $\mathrm{CV}$ global y en salud, y en los dominios físico, psicológico medio ambiente al compararlos con estudiantes inactivos. Esto sugiere la necesidad de promover acciones orientadas hacia la práctica de AF por parte de las instituciones de educación superior y con ello influir sobre la CV de sus estudiantes. Para la implementación de futuros estudios se recomienda un análisis de cada una de las preguntas correspondientes a cada dominio, con el objetivo de conocer cuál o cuáles serían los factores con mayor influencia sobre la autopercepción de $\mathrm{CV}$ en población universitaria.

\section{Limitaciones del estudio}

Una de las limitaciones de esta investigación corresponde a la utilización de una muestra pequeña en relación a la población universitaria estudiada. Además, la selección por conveniencia de los participantes puede restringir la validez externa del estudio. Por otro lado, algunos datos fueron obtenidos mediante auto-reporte (AF y CV), lo cual podría ocasionar un sesgo por la sobreestimación de estudiantes con estilos de vida no saludable. A pesar de estas limitaciones, la significancia estadística obtenida en este análisis con un tamaño del efecto moderado, refleja las diferencias observadas en la CV de la muestra estudiada. 
Conflicto de intereses: Los autores declaramos que no existen conflicto de interés de ningún tipo, ni real o potencial sobre los resultados presentados.

\section{Referencias}

1. Celis-Morales C, Salas C, Alduhishy A, Sanzana R, Martínez MA, Leiva A, Diaz X, Martínez C, Álvarez C, Leppe J, Munro CA, Siervo M, Willis ND Socio-demographic patterns of physical activity and sedentary behaviour in Chile: results from the National Health Survey 2009-2010. J Public Health. 2016;38(2):E98-E105. DOI: 10.1093/pubmed/fdv079

2. García - Hermoso A, Saavedra J, Ramírez - Vélez R, Ekelund U, Pozo - Cruz B. Reallocating sedentary time to moderate to - vigorous physical activity but not to light - intensity physical activity is effective to reduce adiposity among youths: a systematic review and meta - analysis. Obesity Rev. 2017;18(9):1088-95. DOI: 10.1111/obr.12552

3. Organización Mundial de la Salud (WHO). Actividad Física. 2018. Disponible en: https://www.who.int/es/newsroom/fact-sheets/detail/physical-activity

4. Celis-Morales C, Salas C, Álvarez C, Aguilar Farías N, Ramírez Campillos R, Leppe J, et al. Un mayor nivel de actividad física se asocia a una menor prevalencia de factores de riesgo cardiovascular en Chile: resultados de la Encuesta Nacional de Salud 2009-2010. Rev Med Chile. 2015;143:1435-43. http://dx.doi.org/10.4067/S0034-98872015001100009

5. Cervera Burriel F, Serrano Urrea R, Vico García C, Milla Tobarra M, García Meseguer MJ. Hábitos alimentarios y evaluación nutricional en una población universitaria. Nutr Hosp.

2013;28:438-46. http://dx.doi.org/10.3305/nh.2013.28.2.6303

6. Núñez-Rivas HP, Campos-Saborío N, Holst-Schumacher I, Alfaro-Mora FV. Las creencias de la docente de educación física sobre la obesidad en la niñez de edad escolar. Revista Electrónica Educare. 2013;17(2):5-30. Disponible en: https://www.scielo.sa.cr/scielo.php?script=sci_arttext\&pid $=$ S1409-42582013000200002

7. Concha-Cisternas Y, Petermann-Rocha F, Garrido-Méndez Á., Díaz-Martínez X, Leiva AM, Salas-Bravo C, Celis-Morales, C. Caracterización de los patrones de actividad física en distintos grupos etarios chilenos. Nutr Hosp 2019. 36(1), 149-158. http://dx.doi.org/10.20960/nh.1942

8. Hidalgo-Rasmussen CA, Martín H-S. Percepción del peso corporal, comportamiento de control de peso y calidad de vida en adolescentes mexicanos estudiantes de secundaria. Revista mexicana de trastornos alimentarios. 2011;2(2):7181. Disponible en: http://www.scielo.org.mx/scielo.php?script=sci_arttext\&pi $\mathrm{d}=\mathrm{S} 2007-15232011000200003$

9. WHOQOL Group, et al. The World Health Organization Quality of life assessment (WHOQOL): Position paper from the World Health Organization. Soc Sci Med 41(10), 14031409

10. Urzúa A, Caqueo-Urízar A. Calidad de vida: Una revisión teórica del concepto. Terapia psicológica. 2012;30(1):61-71.

11. Krägeloh CU, Kersten P, Billington DR, Hsu PH-C, Shepherd D, Land on J, et al. Validation of the WHOQOL-BREF quality of life questionnaire for general use in New Zealand: Confirmatory factor analysis and Rasch analysis. Qual Life
Res. 2013;22(6):1451-7.http://dx.doi.org/10.4067/S071848082012000100006

12. Benítez-Borrego S, Mancho-Fora N, Farràs-Permanyer L, Urzúa-Morales A, Guàrdia-Olmos J. Differential Item Functioning of WHOQOL-BREF in nine Iberoamerican countries. Revista Iberoamericana de Psicología y Salud. 2016;7(2):51-9.https://doi.org/10.1016/j.rips.2016.04.001

13. Guallar-Castillón P, Peralta PS-0, Banegas JR, López E, Rodríguez-Artalejo F. Actividad física y calidad de vida de la población adulta mayor en España. Med Clin. 2004;123(16):606-10. https://doi.org/10.1016/S00257753(04)74616-3

14. Ministerio de Salud (MINSAL). Encuesta Nacional de Salud 2009-2010. Chile; 2010. Disponible en: https://www.minsal.cl/portal/url/item/bcb03d7bc28b64d fe040010165012d23.pdf

15. Arias JAC, Ospina-Franco LC, Eljadue-Alzamora AP. Validez discriminante, convergente/divergente, fiabilidad y consistencia interna, del WHOQOL-bref y el mossf-36 en adultos sanos de un municipio colombiano. Facultad Nacional de Salud Pública: El escenario para la salud pública desde la ciencia. 2015; 33(1), 50-57. Disponible: https://aprendeenlinea.udea.edu.co/revistas/index.php/fns $\mathrm{p} /$ article/view/15865

16. Serón P, Muñoz S, Lanas F. Nivel de Actividad física medida a través del cuestionario internacional de actividad física en población chilena. Rev Med Chile. 2010; 138:1232-1239. http://dx.doi.org/10.4067/S0034-98872010001100004

17. Crespo-Salgado JJ, Delgado-Martín JL, Blanco-Iglesias O, Aldecoa-Landesa S. Guía básica de detección del sedentarismo y recomendaciones de actividad física en atención primaria. Atención Primaria. 2015;47(3):175-83. https://doi.org/10.1016/j.aprim.2014.09.004

18. Hidalgo-Rasmussen CA, Ramírez-López G, Martín H-S. Physical activity, sedentary behavior and quality of life in undergraduate adolescents of Ciudad Guzman, State of Jalisco, Mexico. Ciencia \& saude coletiva. 2013;18(7):1943 52.http://dx.doi.org/10.1590/S1413-81232013000700009

19. Barros R, Madeira F, Nogueira J. Calidad de vida de universitarios del curso de educación física de una universidad pública brasilera. 2017. VIII Iberoamericano Congreso CIUPS. Universidades promotoras de Salud: aprender, investigar y vivir en salud. Universidad de Alicante (España). 2017.

20. Garber CE, Blissmer B, Deschenes MR, Franklin BA, Lamonte MJ, Lee I-M, et al. American College of Sports Medicine position stand. Quantity and quality of exercise for developing and maintaining cardiorespiratory, musculoskeletal, and neuromotor fitness in apparently healthy adults: guidance for prescribing exercise. Med Sci Sports Exerc. 2011;43(7):1334-1359.

21. Goñi E, Infante G. Actividad físico-deportiva, autoconcepto físico y satisfacción con la vida. European Journal of Education and Psychology. 2015;3(2). Disponible en: https://www.redalyc.org/articulo.oa?id=129315468004

22. Bonet J, Parrado E, Capdevila L. Efectos agudos del ejercicio físico sobre el estado de ánimo y la HRV. Rev. int. med. cienc. act. fís. deporte. 2017;17(65): 85-100. DOI: http://dx.doi.org/10.15366/rimcafd2017.65.006

23. Thorén P, Douglas JSFPH, Seals R. Las endorfinas y el ejercicio: Mecanismos fisiológicos e implicaciones clínicas. Red: revista de entrenamiento deportivo. 2011;25(2):5 -18. Disponible 
https://www.revistadeentrenamientodeportivo.com/articul o/las-endorfinas-y-el-ejercicio-mecanismos-fisiologicos-eimplicaciones-clinicas-1613-sa-T57cfb2723544e/

24. Vizzotto MM, Jesus SNd, Martins AC. Saudades de casa: indicativos de depressão, ansiedade, qualidade de vida e adaptação de estudantes universitários. Revista Psicologia e Saúde. http://dx.doi.org/10.20435/pssa.v9i1.469

25. Ferro T, María J. Sentimientos negativos implicados en el desempeño estudiantil en la clínica odontológica. Acta odontológica venezolana. 2004;42(3):187-91. Disponible en:

https://www.actaodontologica.com/ediciones/2004/3/sen timientos_negativos_clinica_odontologica.asp
26. Olivares CS, Lera ML, Bustos ZN. Etapas del cambio, beneficios y barreras en actividad física y consumo de frutas y verduras en estudiantes universitarios de Santiago de Chile. Rev Chil Nutr. 2008; 35:25-35. http://dx.doi.org/10.4067/S0717-75182008000100004

27. Dunsky A, Zach S, Zeev A, Goldbourt U, Shimony T, Goldsmith R, et al. Level of physical activity and anthropometric characteristics in old age-results from a national health survey. European Review of Aging and Physical Activity. 2014;11(2):149. Disponible en: https://link.springer.com/article/10.1007/s11556-0140139-y 